Regards sur l'économie allemande

Bulletin économique du CIRAC

$102 \mid 2011$

Varia

\title{
Société : conditions de vie et valeurs des Allemands
}

\author{
Isabelle Bourgeois
}

\section{OpenEdition}

\section{Journals}

Édition électronique

URL : http://journals.openedition.org/rea/4340

DOI : $10.4000 /$ rea.4340

ISBN : 978-2-8218-1134-8

ISSN : 1965-0787

Éditeur

CIRAC

Édition imprimée

Date de publication : 31 octobre 2011

Pagination : 36-37

ISSN : 1156-8992

Référence électronique

Isabelle Bourgeois, «Société : conditions de vie et valeurs des Allemands 》, Regards sur l'économie allemande [En ligne], 102 I octobre 2011, mis en ligne le 04 novembre 2011, consulté le 15 septembre 2020. URL : http://journals.openedition.org/rea/4340

Ce document a été généré automatiquement le 15 septembre 2020.

(C) CIRAC 


\title{
Société : conditions de vie et valeurs des Allemands
}

\author{
Isabelle Bourgeois
}

1 Récemment, une série d'indicateurs comparatifs européens a alerté l'opinion sur l'état de la société allemande. Le dernier rapport sur l'éducation de l'OCDE reprend sa litanie sur le faible nombre d'étudiants recensés en Allemagne, en tirant la conclusion quelque peu hâtive (voir REA 88/2008) que le niveau de qualification de la population allemande serait particulièrement faible en comparaison internationale. Les statistiques d'Eurostat classent l'Allemagne parmi les pays de l'UE 27 où le risque de pauvreté est élevé : $15 \%$ de la population est menacée (13\% en France). Régulièrement est ainsi évoquée une montée des disparités sociales.

\section{Datenreport 2011}

2 La publication, le 11 octobre dernier, de la $13^{e}$ édition du « rapport sur l'état social de l'Allemagne », le Datenreport 2011, vient à point nommé pour apporter un peu de factualité et permettre de mieux comprendre ces faits ou idées isolés de leur contexte. Cet ouvrage cumule en effet les richesses de son cousin français, les Données sociales de l'Insee, avec les principales données macro-économiques allemandes - donc le contexte dans lequel évoluent les Allemands -, et une analyse sociologique (voir REA 80/2007). Autant dire que ce summum de connaissances est une 'bible' pour comprendre l'Allemagne. Il est réalisé conjointement, depuis 1985, par Destatis, le Wissenschaftszentrum Berlin für Sozialforschung (WZB), le SOEP auprès de l'institut DIW de Berlin, et publié par la Bundeszentrale für politische Bildung (Bonn).

3 Sa genèse repose sur ce constat, que soulignait la préface de l'édition de 2006: "les statistiques officielles sont un préalable indispensable pour une démocratie vivante». Non seulement, elles apportent la connaissance nécessaire à l'action du politique ou à l'activité des acteurs économiques, mais elles s'adressent également à la science et, encore plus, aux citoyens. Aussi scientifique qu'il soit, ce rapport est donc destiné aussi à un large public "afin de replacer les débats émotionnels sur des fondements rationnels", 
ainsi que le rappellait lors de la conférence de presse Thomas Krüger, président de la Bundeszentrale für politische Bildung, centre indépendant dont la mission est d'œuvrer à la formation de citoyens éclairés. De ce fait, le Datenreport 2011 est bien entendu accessible à tous (www.destatis.de).

\section{Travail féminin en hausse}

4 Nous y apprenons ainsi par exemple que $50 \%$ des Allemands de plus de 15 ans vivent de leur travail, ou que $8 \%$ tirent leur revenu de prestations chômage (allocations ou Hartz IV) ou du Bafög, ce prêt aux étudiants. Les femmes sont $42 \%$ à l'ouest ( $45 \%$ à l'est) à tirer leur revenu principal de leur travail en 2008 , contre respectivement $58 \%$ et $54 \%$ des hommes. A l'ouest comme à l'est, le travail féminin a gagné en importance depuis 2000 , augmentant respectivement de 4 points.

\section{Risque de pauvreté fonction de l'âge et du sexe}

5 En 2008 toujours, 15,5\% des Allemands sont concernés par le risque de pauvreté, c'està-dire qu'ils gagnaient moins de $929 €$ par mois après transferts sociaux. Les plus menacés sont les 18 à 24 ans (21,1\%), et parmi eux surtout les jeunes femmes $(24,4 \%)$, suivis par la cohorte des 55 à 64 ans $(18,1 \%$; sans différence notable entre hommes et femmes) et les femmes de 65 ans ou plus. On retrouve là une photographie des écarts de salaires entre hommes et femmes comme des âges de la vie professionnelle : les jeunes la commencent, les seniors la terminent ou l'ont terminée. Comme en France, ce sont également les célibataires qui sont les plus menacés : ils sont $29,3 \%$ (pas de différence sexuée); et, bien sûr, les parents isolés $(37,9 \%)$. Il faut dire que sur ce point, l'Allemagne ressemble à la France : $44 \%$ vivent en couple (mariage), $6 \%$ sont 'pacsés', $43 \%$ sont célibataires et $6 \%$ sont des parents isolés. Les chômeurs sont $62 \%$ à être exposés au risque de pauvreté, contre seulement $6,8 \%$ des actifs occupés et $14,9 \%$ des retraités. Enfin, comme partout, plus les qualifications sont élevées, plus ce risque diminue : les Allemands faiblement qualifiés (ISCED 0-2) sont $23,2 \%$, les moyennement qualifiés, $14,6 \%$, et les hautement qualifiés (ISCED 5-6) seulement 8,1\%.

\section{Satisfaction : clivage est-ouest}

6 Ces différences se reflètent dans la manière dont les Allemands ressentent subjectivement leur situation (prospérité ou bien-être). Là aussi, plus le niveau de qualification et d'emploi est élevé, plus ils estiment que leur standard de vie est « juste/ plus que juste ", et plus ils sont satisfaits de la vie en général. Dans ce ressenti subjectif, les différences est-ouest sont particulièrement nettes. Ainsi, les employés hautement qualifiés/fonctionnaires occupant des fonctions supérieures sont $74 \%$ à l'ouest et seulement $48 \%$ à l'est à considérer leur situation comme "juste/plus que juste " (2010). L'écart est aussi ou presque aussi net dans toutes les autres catégories CSP - sauf chez les apprentis et les étudiants, dont le taux de réponses est respectivement de $75 \%$ et $63 \%$. Voilà qui confirme la haute image de l'apprentissage en Allemagne: un apprenti 'est quelqu'un'... 


\section{Large sentiment d'appartenance à la classe moyenne}

7 Autre information intéressante à relever : le sentiment d'appartenance à une couche sociale. En 2010, 62 \% des Allemands de l'ouest et $51 \%$ des Allemands de l'est pensent appartenir à la classe moyenne ; respectivement $22 \%$ et $39 \%$ à la classe ouvrière, $13 \%$ et $6 \%$ à la classe supérieure et seulement $3 \%$ et $4 \%$ aux classes inférieures. Le ressenti subjectif est plus prononcé à l'est: l'appartenance à la classe ouvrière plonge ses racines dans «l'Etat d'ouvriers et de paysans » que se déclarait officiellement la RDA. A l'ouest, le sentiment d'appartenance à la classe moyenne, qui n'a que peu varié en tendance ces 30 dernières années, correspond à la réalité statistique; et même la légère augmentation du nombre de ceux qui se situent dans les classes inférieures s'explique par le fait que les couches moyennes tendent objectivement à 'fondre' (voir REA 87/2008), les deux extrémités enregistrant une hausse, principalement sous l'effet d'un écart croissant dans les qualifications des Allemands de souche et d'origine immigrée (voir REA 88/2008).

8 Dans l'ensemble, les Allemands se sentent heureux et contents de vivre. La différence entre l'est (6,5 points sur une échelle de 10) et l'ouest $(6,9)$ s'est nettement estompée en 2009. Ce qui les satisfait le plus, c'est leur logement ( 7,6 et 7,7$)$, suivi par leurs loisirs $(6,9$ et 7,1$)$ et leur travail $(6,6$ et 6,7$)$. Ce qu'ils désirent ? Plus d'influence des citoyens sur les décisions des gouvernements (30\% des réponses à l'ouest, $35 \%$ à l'est ; 2008), préservation du calme et de l'ordre ( $27 \%$ des deux côtés) et lutte contre la hausse des prix $(24 \%$ contre $28 \%)$. Enfin, loin derrière seulement vient la préservation du droit à la libre expression ( $21 \%$ contre $10 \%$ ). Il est vrai que, dans ce domaine, l'Allemagne offre quelques garanties.

INDEX

Mots-clés : société, conditions de vie, femme, groupe social, marché du travail, emploi, pauvreté, niveau de vie, satisfaction, classe moyenne 